08

\title{
Направление диффузионного потока галлия при осаждении на поверхность с регулярными массивами отверстий
}

\author{
() В.Г. Дубровский \\ Санкт-Петербургский государственный университет, Санкт-Петербург, Россия \\ E-mail: dubrovskii@mail.ioffe.ru
}

Поступило в Редакцию 12 марта 2021 r.

В окончательной редакции 12 марта 2021 г.

Принято к публикации 22 марта 2021 г.

\begin{abstract}
Автокаталитический рост нитевидных нанокристаллов $\mathrm{GaAs}$ и $\mathrm{GaP}$ часто осуществляется на поверхностях $\mathrm{SiO}_{x} / \mathrm{Si}(111)$ с массивами литографически подготовленных отверстий. Капли Ga создаются в процессе предварительного осаждения в отсутствие потока As. Ранее считалось, что диффузионный поток Ga направлен с поверхности маски в отверстия. Нами показано, что направление диффузионного потока может быть различным в зависимости от параметров роста. Модель применима для описания времен инкубации капель и позволяет объяснить длительную задержку нуклеации капель и нитевидных нанокристаллов.
\end{abstract}

Ключевые слова: поверхностная диффузия, массивы отверстий, оксидный слой кремния, капли галлия.

DOI: 10.21883/PJTF.2021.12.51063.18765

Полупроводниковые нитевидные нанокристаллы (ННК), в особенности III-V ННК, являются перспективной платформой как для фундаментальных исследований в области нанофотоники и наноэлектроники, так и для приборных приложений [1]. Эффективная релаксация упругих напряжений за счет огромного аспектного соотношения позволяет подавить формирование дислокаций несоответствия в гетероструктурах на основе III-V ННК при их росте на подложке кремния [2,3], что невозможно в тонких пленках и даже в наноостровках Странского-Крастанова [4]. Одним из интереснейших свойств III-V ННК является возможность их формирования в вюрцитной кристаллической фазе [5]. Большинство III-V ННК выращивается по механизму пар-жидкость-кристалл (ПЖК) [6], исследованию которого посвящено большое число работ (см, например, обзор [7]). В качестве металлических катализаторов роста ПЖК чаще всего используются $\mathrm{Au}[6,8,9]$ или металлы группы III (Ga [10-12] или In [13]), в последнем случае рост ПЖК называют автокаталитическим.

Автокаталитический рост позволяет не только избежать возможного загрязнения $\mathrm{Au}$, но и выращивать III-V HНК методом молекулярно-пучковой эпитаксии (МПЭ) в регулярных массивах литографически подготовленных отверстий в оксидном слое $\mathrm{SiO}_{x}$ на поверхности $\mathrm{Si}(111)$. Капли создаются путем предварительного осаждения $\mathrm{Ga}$ на поверхность $\mathrm{SiO}_{x} / \mathrm{Si}(111)$; $\mathrm{Ga}$ собирается в отверстиях за счет поверхностной диффузии. Рост по механизму селективной эпитаксии во многих случаях имеет характер автокаталитического [14]. Обычно предполагается [12-14], что диффузионный поток Ga направлен с поверхности маски в отверстия, однако это противоречит некоторым данным по медленному формированию капель и существенным задержкам нуклеации
ННК [12]. В настоящей работе мы исследуем процесс теоретически и определяем критерий для диффузии $\mathrm{Ga}$ в отверстия или из них, что также позволяет найти зависимости времен инкубации и роста капель от радиуса отверстий и расстояния между ними.

Геометрия задачи показана на рис. 1. Расстояние между центрами отверстий $P=L+2 R$, где $L-$ расстояние между границами соседних отверстий, $R$ - радиус отверстий. Двумерное стационарное уравнение диффузии для концентрации адатомов Ga на поверхности оксидной маски $n$ имеет вид [7]:

$$
D \Delta n+J-\frac{n}{\tau}=0
$$

Здесь $D-$ коэффициент диффузии $\mathrm{Ga}, J-$ газовый поток $\mathrm{Ga}, \tau$ - характерное время десорбции (рис. 1). В предположении симметрии по углу общее решение (1) есть

$$
n=J \tau+A I_{0}(r / \lambda)+B K_{0}(r / \lambda),
$$

где $\lambda=\sqrt{D \tau}-$ диффузионная длина адатомов $\mathrm{Ga}$, а $I_{0}(x)$ и $K_{0}(x)$ обозначают модифицированные функции Бесселя нулевого порядка. Из соображений симметрии граничное условие между отверстиями (при $r=0)$ соответствует обнулению диффузионного потока: $(d n / d r)_{r=0}=0$, откуда $B=0$.

В дальнейшем мы предполагаем пространственно однородное значение поверхностной плотности адатомов $\mathrm{Ga}$ в отверстия $n_{h}$, что справедливо при $R \ll P$. Тогда полный ток адатомов $\mathrm{Ga}$ внутрь отверстия или из отверстия на поверхность маски есть

$$
\frac{d N_{h}}{d t}=\pi R^{2}\left(J-\frac{n_{h}}{\tau_{*}}\right), \quad \frac{1}{\tau_{*}}=\frac{1}{\tau_{h}}+\frac{1}{\tau_{g}} .
$$

Характерная скорость стока адатомов $\mathrm{Ga} 1 / \tau_{*}$ равна сумме скорости десорбции с поверхности кремния 


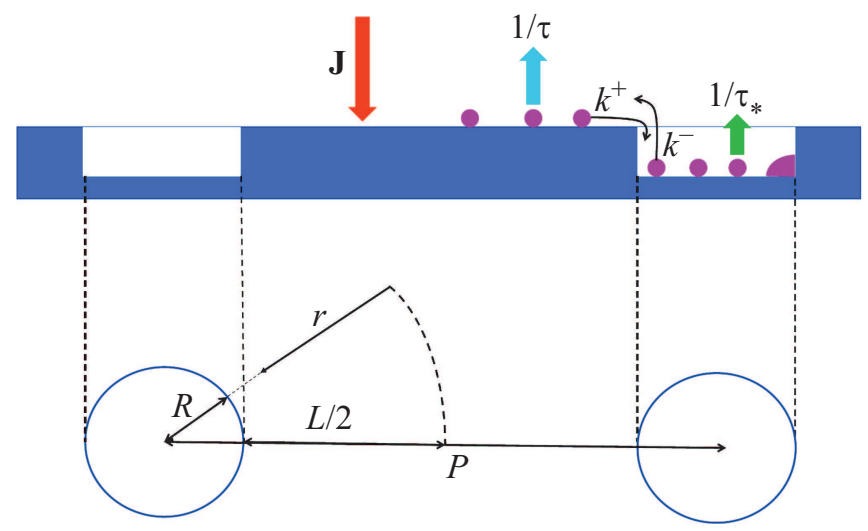

Рис. 1. Иллюстрация модели и основных параметров.

$1 / \tau_{h}$ и скорости роста капли в линейном приближении $1 / \tau_{g}$ [15]. На стадии инкубации (до момента нуклеации капли) имеем просто $1 / \tau_{*}=1 / \tau_{h}$. Из условия непрерывности потока на границе отверстия следует

$$
2 \pi R D\left(\frac{d n}{d r}\right)_{r=L / 2}=\pi R^{2}\left(J-\frac{n_{h}}{\tau_{*}}\right) .
$$

Для определения неизвестного значения $n_{h}$ мы привлекаем еще одно граничное условие, следующее из соображений кинетического баланса [16] и использованное ранее в ряде работ по моделированию роста HНK [17-19]:

$$
-D\left(\frac{d n}{d r}\right)_{r=L / 2}=k^{+} n(r=L / 2)-k^{-} n_{h} .
$$

Здесь $k^{+}$и $k^{-}$- константы скорости переходов адатомов с поверхности оксидного слоя в отверстие и наоборот (рис. 1). Из (4) и (5) находим

$$
n_{h}=J \tau_{*} \frac{k^{+} \tau+V}{k^{-} \tau_{*}+V}, V=\frac{R}{2}\left[1+\frac{k^{+} \tau}{\lambda} \frac{I_{0}(L / 2 \lambda)}{I_{1}(L / 2 \lambda)}\right],
$$

где $I_{1}(x)$ - модифицированная функция Бесселя первого порядка. В линейном приближении как время инкубации, так и характерное время роста капли Ga в отверстии обратно пропорциональны поверхностной плотности адатомов $\mathrm{Ga}$

$$
t_{\text {inc }} \propto \frac{1}{n_{h}}, \quad t_{\text {growth }} \propto \frac{1}{n_{h}} .
$$

Исследование (6) приводит к следующим выводам. Из (6) и (3) легко получить $d N_{h} / d t \propto k^{-} \tau_{*}-k^{+} \tau$, $d n_{h} / d R \propto k^{-} \tau_{*}-k^{+} \tau \quad$ и $d n_{h} / d L \propto k^{+} \tau-k^{-} \tau_{*}$. Следовательно, возможны два режима диффузии адатомов Ga. При $k^{-} \tau_{*}-k^{+} \tau>0$ имеем $d N_{h} / d t>0, d n_{h} / d R>0$ и $d n_{h} / d L<0$. Диффузионный поток $\mathrm{Ga}$ направлен из отверстий на поверхность оксидной маски. Плотность адатомов $\mathrm{Ga}$ в отверстиях возрастает при увеличении их радиуса и убывает при увеличении расстояния между отверстиями. В соответствии с (7) капли Ga формируются быстрее в отверстиях большего радиуса и для более плотных массивов отверстий. В противоположном случае $k^{-} \tau_{*}-k^{+} \tau<0$ имеем $d N_{h} / d t<0$, $d n_{h} / d R<0$ и $d n_{h} / d L>0$. Диффузионный поток Ga направлен внутрь отверстий, как и предполагалось ранее [12-14]. Плотность адатомов $\mathrm{Ga}$ выше в отверстиях меньшего радиуса и при больших расстояниях между отверстиями. Поэтому отверстия малого радиуса и плотности заполняются быстрее. Если диффузионный поток направлен в отверстия, то замедление формирования капель в более плотных массивах отверстий объясняется перекрытием зон питания различных отверстий [13].

Зависимости плотности адатомов Ga от радиуса отверстий $R$ при фиксированном $P$ изображены на рис. 2 . На рис. 3 приведены аналогичные зависимости от $P$ при фиксированном значении $R$. Рис. 2 и 3 наглядно демонстрируют различие между двумя режимами поверхностной диффузии $\mathrm{Ga}$, приводящими к противоположным типам зависимости плотности адатомов от размера и расстояния между отверстиями.

Как уже указывалось, направление диффузионного потока с поверхности оксидной маски в отверстия кажется естественным, в особенности для глубоких отверстий с высокими значениями барьеров Эрлиха-Швобеля для диффузии адатомов из отверстий [20]. С другой стороны, адатомы $\mathrm{Ga}$ должны быть связаны гораздо слабее с поверхностью $\mathrm{SiO}_{x}$ в сравнении с $\mathrm{Si}$ на дне отверстий. Именно это свойство и обеспечивает селективность маски. Следовательно, должно быть выполнено неравенство $\tau_{*}>\tau$ или даже $\tau_{*} \gg \tau$, что может привести к выполнению неравенства $k^{-} \tau_{*}-k^{+} \tau>0$

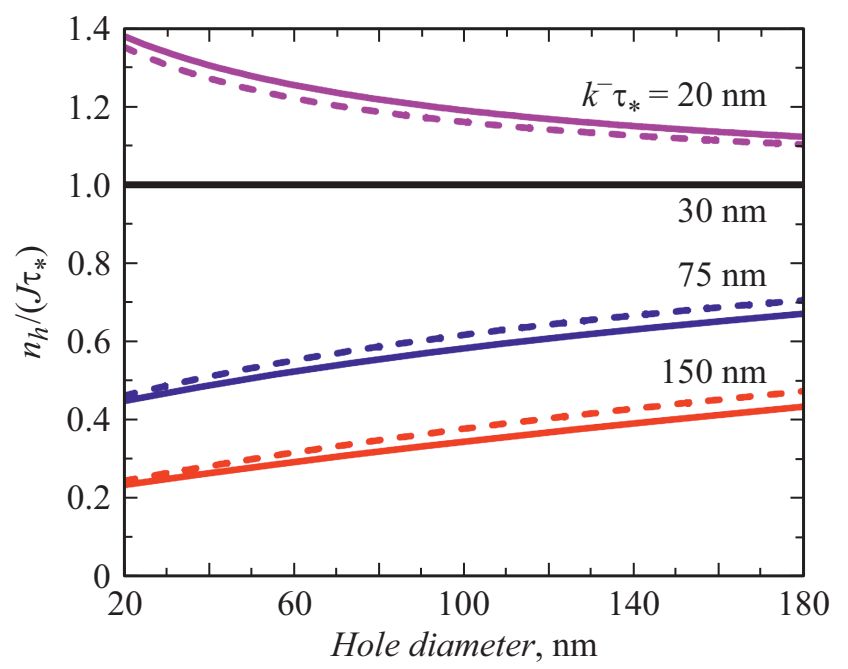

Рис. 2. Безразмерная плотность адатомов $\mathrm{Ga}$ в отверстии $n_{h} /\left(J \tau_{*}\right)$ в зависимости от диаметра отверстий $2 R$, полученная из уравнения (6) при фиксированном $P=520 \mathrm{~nm}$, $k^{+} \tau=30 \mathrm{~nm}, \lambda=300 \mathrm{~nm}$ (сплошные линии) и $50 \mathrm{~nm}$ (штриховые линии) для различных значений $k^{-} \tau_{*}$. 


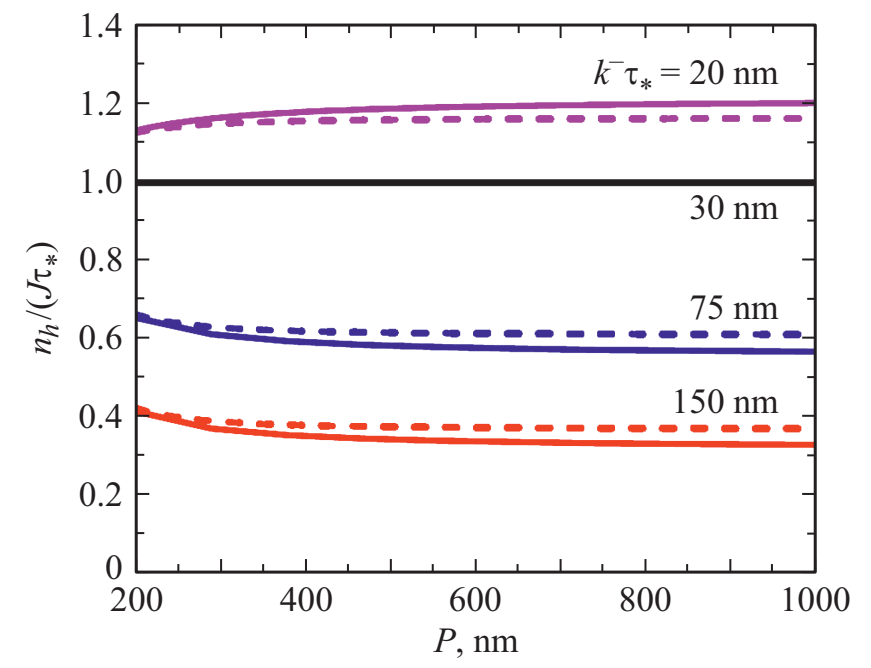

Рис. 3. То же, что на рис. 2 , в зависимости от расстояния между отверстиями $P$ при фиксированном $R=50 \mathrm{~nm}$.

даже при $k^{-}<k^{+}$. Поэтому осуществление необычного режима формирования капель $\mathrm{Ga}$, при котором диффузионный поток направлен из отверстий, весьма вероятно. Данный эффект позволяет объяснить длительные времена инкубации капель и нуклеации GaAs в отверстиях при автокаталитическом МПЭ-росте HНК GaAs в массивах отверстий на поверхностях $\mathrm{SiO}_{x} / \mathrm{Si}(111)[12]$.

В заключение отметим, что в работе исследованы режимы поверхностной диффузии адатомов $\mathrm{Ga}$ на процессированных поверхностях $\mathrm{SiO}_{x} / \mathrm{Si}(111)$ и показана возможность диффузии из отверстий на поверхность оксидной макси, что приводит к необычной возрастающей зависимости скорости формирования капель $\mathrm{Ga}$ от радиуса отверстий и их плотности. При этом значительная часть атомов $\mathrm{Ga}$ испаряется с поверхности маски, что и приводит к задержке формирования капель. Предложенная модель не является специфической для системы $\mathrm{Ga}-\mathrm{SiO}_{x} / \mathrm{Si}(111)$ и может применяться для моделирования нуклеации и роста различных капель в отверстиях, а также роста наноструктур методом селективной МПЭ.

\section{Благодарности}

Автор признателен F. Glas, который получил соответствующие решения в одномерном случае, за полезные обсуждения.

\section{Финансирование работы}

Работа выполнена при поддержке грантов Российского фонда фундаментальных исследований № 20-52-16301, 20-02-00351 и 18-02-40006.

\section{Конфликт интересов}

Автор заявляет, что у него нет конфликта интересов.

\section{Список литературы}

[1] A. Zhang, G. Zheng, C.M. Lieber, Nanowires: building blocks for nanoscience and nanotechnology (Springer, 2016).

[2] F. Glas, Phys. Rev. B, 74, 121302(R) (2006). DOI: $10.1103 /$ PhysRevB.74.121302

[3] V.G. Dubrovskii, N.V. Sibirev, X. Zhang, R.A. Suris, Cryst. Growth Design, 10, 3949 (2010). DOI: 10.1021/cg100495b

[4] G.E. Cirlin, V.G. Dubrovskii, V.N. Petrov, N.K. Polyakov, N.P. Korneeva, V.N. Demidov, A.O. Golubok, S.A. Masalov, D.V. Kurochkin, O.M. Gorbenko, N.I. Komyak, V.M. Ustinov, A.Yu. Egorov, A.R. Kovsh, M.V. Maximov, A.F. Tsatusul'nikov, B.V. Volovik, A.E. Zhukov, P.S. Kop'ev, Zh.I. Alferov, N.N. Ledentsov, M. Grundmann, D. Bimberg, Semicond. Sci. Technol., 13, 1262 (1998). DOI: 10.1088/0268-1242/13/11/005

[5] M.H. Sun, E.S.P. Leong, A.H. Chin, C.Z. Ning, G.E. Cirlin, Yu.B. Samsonenko, V.G. Dubrovskii, L. Chuang, C. ChangHasnain, Nanotechnology, 21, 335705 (2010). DOI: 10.1088/0957-4484/21/33/335705

[6] R.S. Wagner, W.C. Ellis, Appl. Phys. Lett., 4, 89 (1964). DOI: $10.1063 / 1.1753975$

[7] V.G. Dubrovskii, F. Glas, in: Fundamental properties of semiconductor nanowires, ed. by N. Fukata, R. Rurali (Springer, 2021), p. 3. DOI: 10.1007/978-981-15-9050-4_1

[8] V.G. Dubrovskii, I.P. Soshnikov, G.E. Cirlin, A.A. Tonkikh, Yu.B. Samsonenko, N.V. Sibirev, V.M. Ustinov, Phys. Status Solidi B, 241, R30 (2004). DOI: 10.1002/pssb.200409042

[9] Г.Э. Цырлин, В.Г. Дубровский, Н.В. Сибирев, И.П. Сошников, Ю.Б. Самсоненко, А.А. Тонких, В.М. Устинов, ФТП, 39 (5), 587 (2005).

[10] C. Colombo, D. Spirkoska, M. Frimmer, G. Abstreiter, A. Fontcuberta i Morral, Phys. Rev. B, 77, 155326 (2008). DOI: 10.1103/PhysRevB.77.155326

[11] S. Plissard, G. Larrieu, X. Wallart, P. Caroff, Nanotechnology, 22, 275602 (2011). DOI: 10.1088/0957-4484/22/27/275602

[12] J. Vukajlovic-Plestina, W. Kim, V.G. Dubrovskii, G. Tütüncüoğlu, M. Lagier, H. Potts, M. Friedl, A. Fontcuberta i Morral, Nano Lett., 17, 4101 (2017). DOI: 10.1021/acs.nanolett.7b00842

[13] M.T. Robson, V.G. Dubrovskii, R.R. LaPierre, Nanotechnology, 26, 465301 (2015). DOI: $10.1088 / 0957-4484 / 26 / 46 / 465301$

[14] Q. Gao, V.G. Dubrovskii, P. Caroff, J. Wong-Leung, L. Li, Y. Guo, L. Fu, H.H. Tan, C. Jagadish, Nano Lett., 16, 4361 (2016).

DOI: $10.1021 /$ acs.nanolett.6b01461

[15] V.G. Dubrovskii, Phys. Status Solidi B, 171, 345 (1992). DOI: $10.1002 / \mathrm{pssb} .2221710206$

[16] P. Hänggi, P. Talkner, M. Borkovec, Rev. Mod. Phys., 62, 251 (1990). DOI: 10.1103/RevModPhys.62.251

[17] P. Krogstrup, H.I. Jørgensen, E. Johnson, M.H. Madsen, C.B. Sørensen, A. Fontcuberta i Morral, M. Aagesen, J. Nygård, F. Glas, J. Phys. D: Appl. Phys., 46, 313001 (2013). DOI: $10.1088 / 0022-3727 / 46 / 31 / 313001$ 
[18] V.G. Dubrovskii, Yu.Yu. Hervieu, J. Cryst. Growth, 401, 431 (2014). DOI: 10.1016/j.jcrysgro.2014.01.015

[19] Yu.Yu. Hervieu, J. Cryst. Growth, 493, 1 (2018). DOI: j.jcrysgro.2018.04.012

[20] R.L. Schwoebel, J. Appl. Phys., 37, 3682 (1966). DOI: $10.1063 / 1.1707904$ 